\title{
SIRT3 diminishes inflammation and mitigates endotoxin-induced acute lung injury
}

\author{
Deepali Kurundkar, ${ }^{1}$ Ashish R. Kurundkar, ${ }^{2}$ Nathaniel B. Bone, ${ }^{1}$ Eugene J. Becker Jr., ${ }^{1}$ Wanqu Liu, ${ }^{1}$
} Balu Chacko, ${ }^{2}$ Victor Darley-Usmar, Jaroslaw W. Zmijewski, ${ }^{2}$ and Victor J. Thannickal'

'Department of Medicine and 2Department of Pathology, University of Alabama at Birmingham, Birmingham, Alabama, USA.

Acute lung injury (ALI) is characterized by exuberant proinflammatory responses and mitochondrial dysfunction. However, the link between mitochondrial dysfunction and inflammation in ALI is not well understood. In this report, we demonstrate a critical role for the mitochondrial NAD+dependent deacetylase, sirtuin-3 (SIRT3), in regulating macrophage mitochondrial bioenergetics, ROS formation, and proinflammatory responses. We found that SIRT3 expression was significantly diminished in lungs of mice subjected to LPS-induced ALI. SIRT3-deficient mice (SIRT3-1-) develop more severe ALI compared with wild-type controls (SIRT3 ${ }^{+/+}$). Macrophages obtained from SIRT3-/- mice show significant alterations in mitochondrial bioenergetic and redox homeostasis, in association with a proinflammatory phenotype characterized by NLRP3 inflammasome activation. The SIRT3 activator viniferin restored macrophage bioenergetic function in LPS-treated macrophages. Viniferin also reduced NLRP3 activation and the production of proinflammatory cytokines, effects that were absent in SIRT3-/- macrophages. In-vivo administration of viniferin reduced production of inflammatory mediators TNF- $\alpha$, MIP-2, IL-6, IL-1 1 , and HMGB1, and diminished neutrophil influx and severity of endotoxin-mediated ALI; this protective effect of vinferin was abolished in SIRT3 ${ }^{-/-}$mice. Taken together, our results show that the induction/ activation of SIRT3 may serve as a new therapeutic strategy in ALI by modulating cellular bioenergetics, controlling inflammatory responses, and reducing the severity of lung injury.

Authorship note: JWZ and VJT contributed equally to this work.

Conflict of interest: The authors have declared that no conflict of interest exists.

License: Copyright 2019, American Society for Clinical Investigation.

Submitted: February 26, 2018 Accepted: December 5, 2018 Published: January 10, 2019

\section{Reference information:} JCI Insight. 2019;4(1):e120722. https://doi.org/10.1172/jci. insight.120722.

\section{Introduction}

Acute lung injury (ALI) and the acute respiratory distress syndrome (ARDS) are characterized by activation of neutrophils, macrophages, alveolar capillary membrane barrier dysfunction/disruption, and edema that frequently lead to respiratory failure (1). Sepsis remains the primary risk factor for the development of ALI/ARDS. In addition to microbial infections, sterile inflammatory conditions, including shock and trauma may also lead to ARDS $(1,2)$. In this setting, the cytokine storm and host tissue-derived damage-associated molecular pattern proteins (DAMPs) including high-mobility group box-1 (HMGB1) play an important role in organ injury (3). Despite significant progress in clinical care, ARDS continues to carry a high mortality rate $(2,4)$. Previous therapeutic strategies that have targeted specific cytokines have not been effective in reducing the ARDS risk or mortality from sepsis $(5,6)$

Innate immune responses are assigned to eradicate microbial infections $(7,8)$; however, exaggerated or dysregulated inflammatory responses may contribute to organ injury and high mortality among critically ill subjects $(6,9,10)$. Importantly, the biomarkers of mitochondrial dysfunction correlate with the severity of lung injury and mortality in sepsis-related ARDS $(11,12)$. Recent studies indicate that mitochondrial dysfunction also occurs in circulating monocytes and this is indicative of poor prognosis in critically ill subjects $(13,14)$. A few preclinical studies have shown that preservation of mitochondrial function reduces the severity of sepsis in endotoxin-induced ALI $(15,16)$. These studies suggest that modulation of mitochondrial function may be beneficial in ARDS.

Sirtuins are histone deacetylases involved in regulating metabolic homeostasis in many cell populations including immune cells (17-19). In mammals, seven known sirtuins (SIRT1-7) are characterized by distinct subcellular localization and substrate specificity (20). Recent studies have suggested that mitochondrial SIRT3 
is involved in regulating immune cell function and the development of inflammatory organ injury (21-23); however, whether SIRT3 induction/activation may provide protection from ALI/ARDS is unknown. Given the importance of SIRT3 in regulating mitochondrial function $(19,24)$, we hypothesized that SIRT3 induction/activation will prevent macrophage metabolic reprogramming and restrain its proinflammatory phenotype, thus diminishing endotoxin-induced ALI.

\section{Results}

SIRT3 expression is diminished in LPS-induced ALI. SIRT3 is an $\mathrm{NAD}^{+}$-dependent deacetylase that is primarily localized to the mitochondria $(25,26)$, and its deficiency has been linked to modulation of mitochondrial function and redox homeostasis $(27,28)$. The role of SIRT3 in ALI is unclear. In the first set of experiments, we determined whether SIRT3 expression levels were altered in the lungs of LPS-treated mice. Endotoxin-induced ALI is a well-characterized model of nonlethal ALI mediated by proinflammatory activation of immune cells and increased vascular permeability, leading to pulmonary edema (29). Neutrophil influx and accumulation of cellular debris within alveolar spaces, in association with a thickened septum, were observed in mice subjected to LPS-induced ALI (Figure 1A). These characteristic features of ALI were associated with reduced levels of the SIRT3 in the lung, as evidenced by immunofluorescent staining of lung sections 24 hours after LPS exposure (Figure 1B). We also observed lower levels of the mitochondrial marker, COX IV, and enhanced mitochondrial fragmentation (Figure 1, B and C). Western blot analyses confirmed that SIRT3 was significantly reduced in both whole-lung homogenates and in the mitochondrial fractions of LPS-treated mice (Figure 1D). This decreased level of SIRT3 in LPS-treated mice was also evident at the mRNA level in lung homogenates (Figure 1D). SIRT3 expression was also reduced in flow-sorted alveolar macrophages from bronchoalveolar lavage (BAL) fluids, i.e., 24 hours following LPS exposure in vivo (Figure 1E), as well as in isolated alveolar macrophages stimulated with LPS for 24 hours ex vivo (Figure 1F). Together, these findings indicate that the mitochondrial SIRT3 is downregulated in alveolar macrophages and lungs of mice subjected to LPS-induced ALI. To confirm our findings in LPS-induced ALI, we induced pneumonia in mice by administering live bacteria (Pseudomonas aeruginosa) by intratracheal (i.t.) administration. We found that SIRT3 was markedly downregulated within 4 hours after bacterial challenge; this resulted in lower levels of superoxide dismutase-2 (SOD2) acetylation, which is largely accounted for by a decrease in total SOD2 levels (Supplemental Figure 1, A-C; supplemental material available online with this article; https://doi.org/10.1172/jci.insight.120722DS1). In addition, we found that SIRT3 deficiency did not adversely affect bacterial clearance (Supplemental Figure 1D).

SIRT3 deficiency exacerbates injury in an experimental model of endotoxin-induced ALI. To test the importance of reduced expression of SIRT3 in experimental ALI, the severity of injury was measured in LPS-treated $S_{R T 3^{+/+}}$and SIRT3 ${ }^{-/-}$mice. Severity of ALI was determined using several indices of ALI including alveolar protein accumulation and levels of cytokines in BAL fluids. H\&E-stained lung sections were prepared to assess lung alveolar architecture and neutrophil recruitment. Compared with wild-type mice, SIRT3 deficiency resulted in more extensive accumulation of neutrophils and cellular debris, along with an increase in the levels of BAL protein, measured 24 hours after LPS exposure (Figure 2, A-D). We also observed a significant increase in proinflammatory cytokines, in particular TNF- $\alpha$, MIP-2, and IL-18, in LPS-treated SIRT3 $^{-1-}$ compared with wild-type mice (Figure 2, E-H). These results indicate that SIRT3 deficiency exacerbates LPS-mediated inflammation and increases severity of ALI.

SIRT3 deficiency augments mitochondrial ROS formation and macrophage proinflammatory responses. Next, we tested whether SIRT3 deficiency in macrophages modulates its bioenergetic function and proinflammatory responses. Mitochondrial ROS production was determined using MitoSOX, a probe capable of measuring mitochondrial superoxide. Flow cytometry results indicated an increase in the MitoSOX fluorescence in $\mathrm{SIRT3}^{-1-}$ macrophages with LPS stimulation, as compared with wild-type counterparts (Figure 3, A and B). Additionally, the cellular bioenergetic profile of $S I R T 3^{+/+}$and $S I R T 3^{-/-}$macrophages was analyzed. In $S I R T 3^{-1-}$ macrophages, maximal oxygen capacity (oxygen consumption rate [OCR]) and reserve capacity were decreased, while proton leak was increased (Figure 3C). These data support a role for SIRT3 in maintaining mitochondrial bioenergetic efficiency in macrophages.

Next, we determined whether SIRT3 deficiency alters the proinflammatory responses of macrophages to LPS. SIRT3 ${ }^{-/-}$macrophages responded more robustly to LPS challenge, with a greater increase in TNF- $\alpha$ production when compared with wild-type cells (Figure 4A). Viniferin is a naturally occurring resveratrol 
A

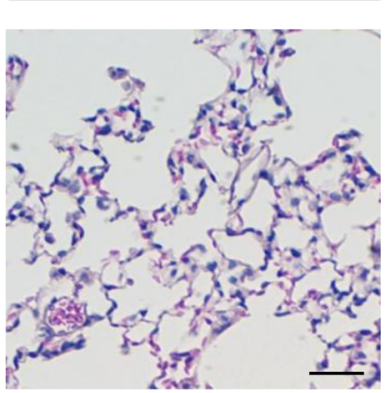

LPS

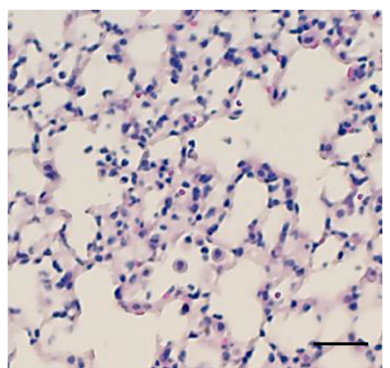

B
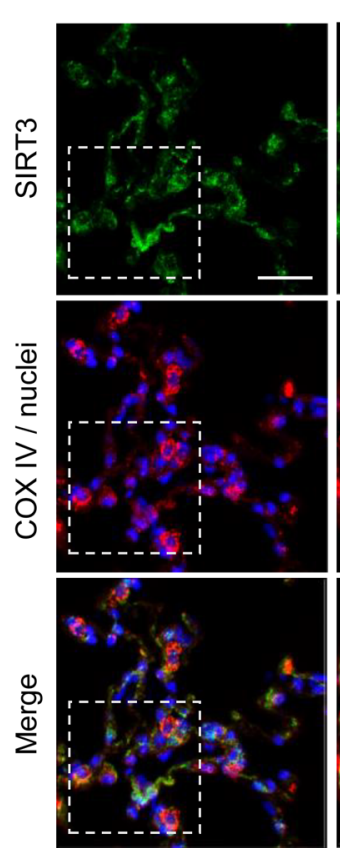

Control

C

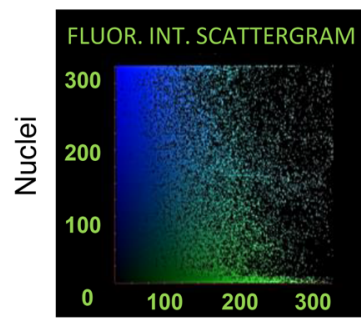

SIRT3

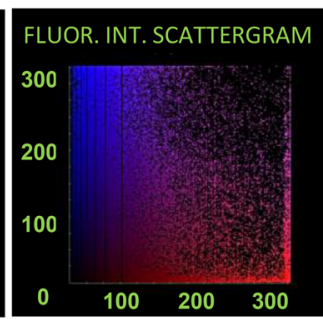

COX IV

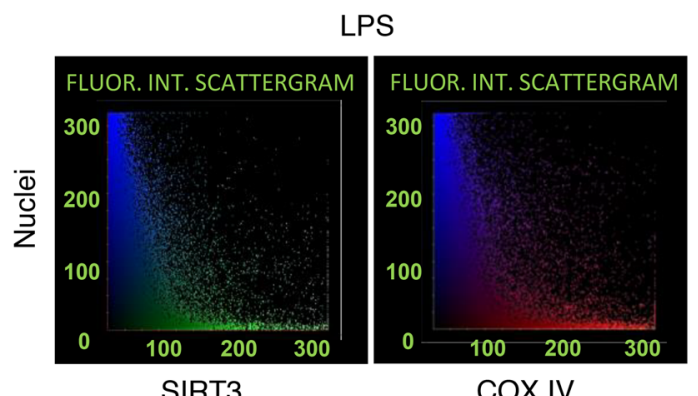

Control
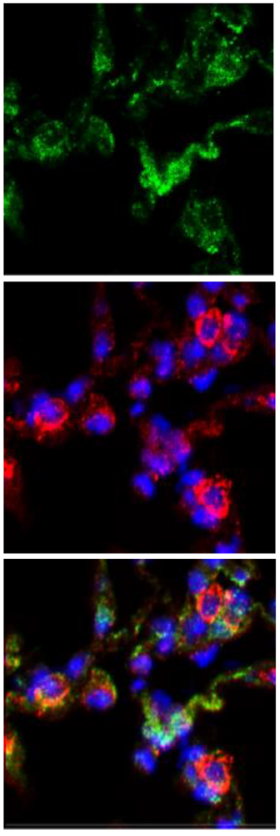

D
LPS
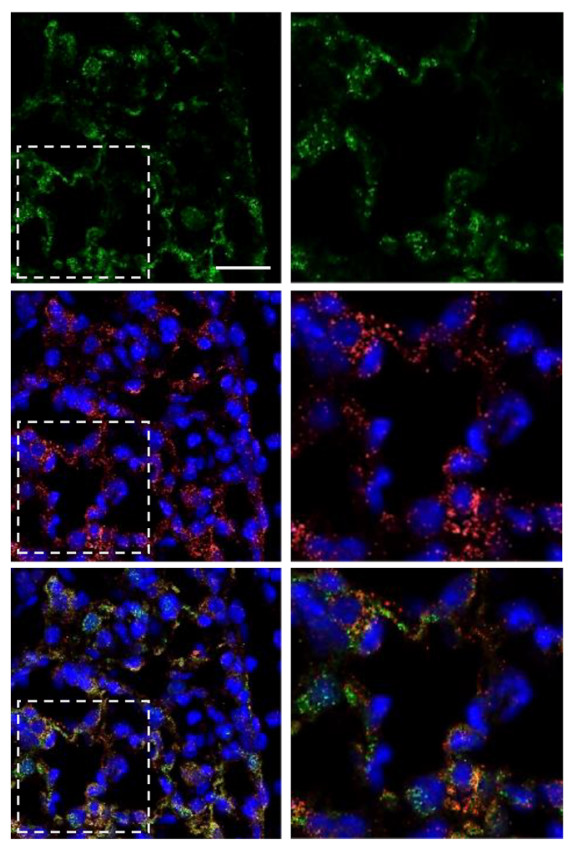
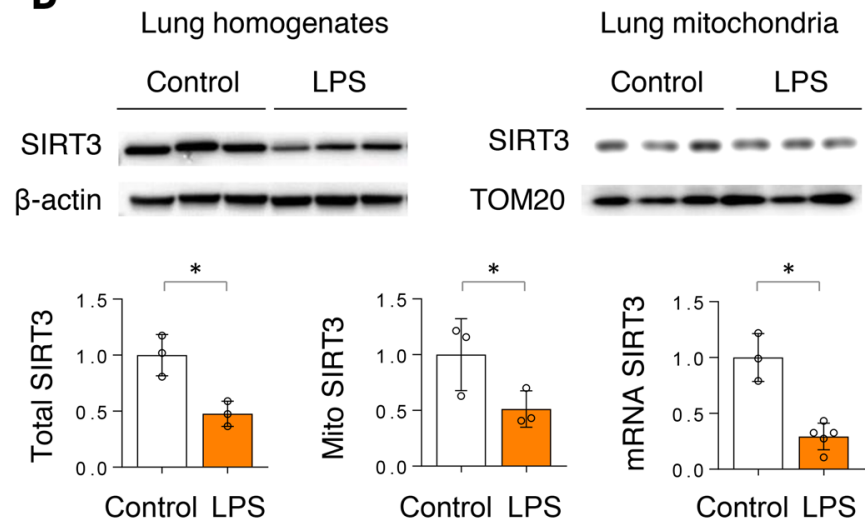

E

Alveolar macrophages

F
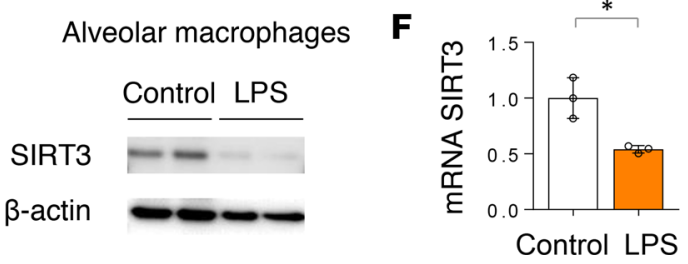

Figure 1. SIRT3 expression is diminished in lungs and alveolar macrophages from mice subjected to endotoxin-induced ALI. (A) Representative images show H\&E staining of lung sections from control and mice treated with LPS (3 mg/kg, i.t.) for 24 hours. Scale bars: $10 \mu \mathrm{m}$. (B) Representative images indicate SIRT3 (green) and COX IV (red) fluorescent staining in lung sections of indicated groups of mice. Right panels depict regions marked by dashed boxes. Scale bars: $10 \mu \mathrm{m}$. (C) Representative scattergrams showing fluorescence intensity from images displayed in B. (D and E) Representative Western blots of SIRT3 in lung homogenates, lung mitochondrial fractions (D), and alveolar macrophages (E) from control or LPS-treated mice. SIRT3 mRNA levels were also determined in lung tissues (D), or (F) alveolar macrophages treated with or without LPS for 24 hours. Data presented as mean \pm SD $(n=3-5)$. ${ }^{*} P<0.05$ (Student's $t$ test).

dimer that has been shown to activate SIRT3 in diverse cell populations $(30,31)$. We found that viniferin increases expression of SIRT3 in peritoneal macrophages (Figure 4B). Pretreatment with viniferin significantly reduces LPS-induced TNF- $\alpha$ production in wild-type, but not SIRT3-1- cells (Figure 4C). In addition to TNF- $\alpha$ production, exposure to LPS is known to activate the NLRP3 inflammasome (32). We found that NLRP3 activation occurs to a greater extent in SIRT3-1- macrophages (Figure 4D). Importantly, viniferin diminishes LPS-induced NLRP3 expression in wild-type, but not SIRT3 ${ }^{-1-}$ macrophages (Figure 4E). 
A

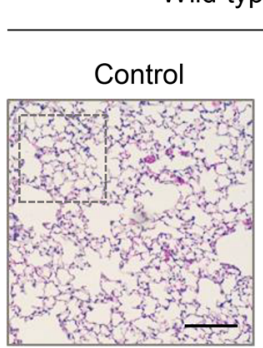

Wild-type $\left(S I R T 3^{+/+}\right)$

B

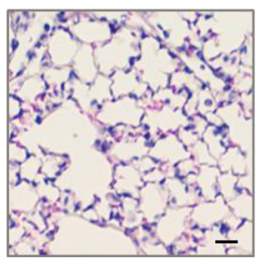

LPS
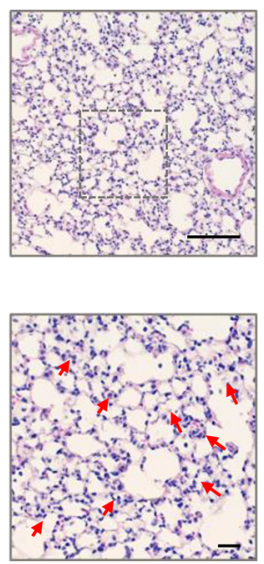

$\operatorname{SIRT3} \mathrm{KO}\left(\mathrm{SIRT}^{-}-\right)^{-}$
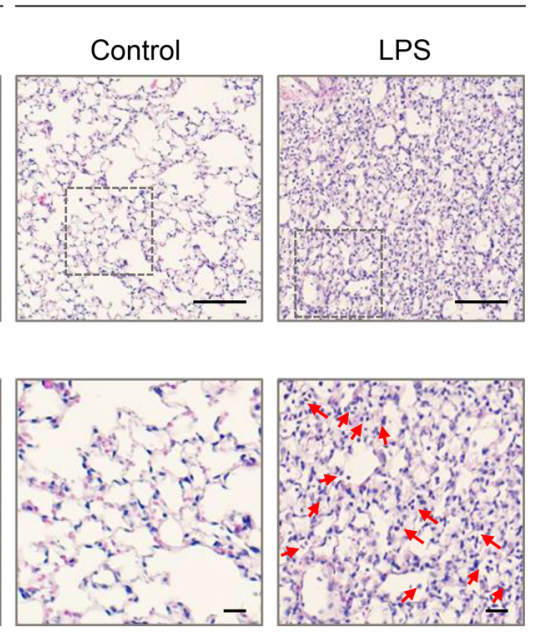

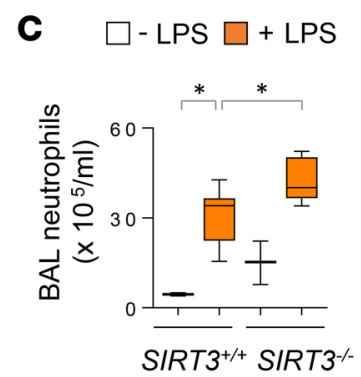

$\mathbf{F}$

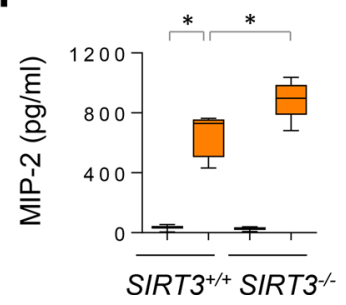

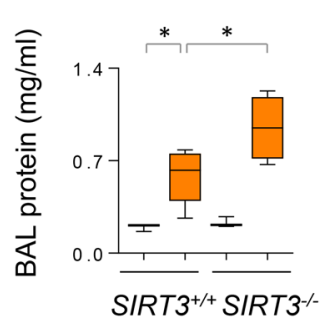

G

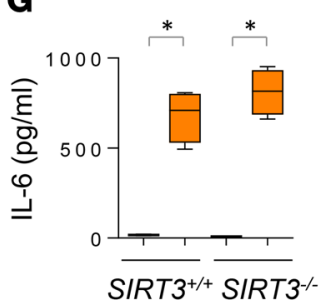

$\mathbf{E}$

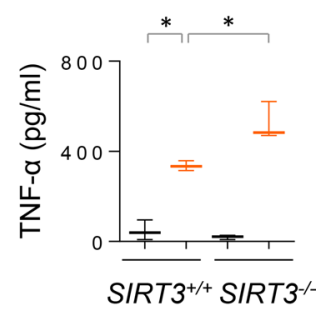

H

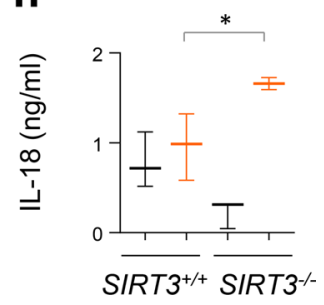

Figure 2. SIRT3 deficiency exacerbates inflammation in lungs of LPS-treated mice. (A and B) Representative images

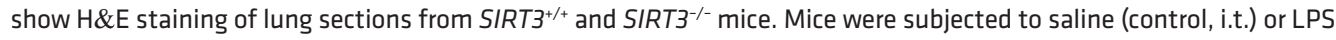
(3 mg/kg, i.t.) for 24 hours. Scale bars: $100 \mu \mathrm{m}$. Lower panel showing regions of images indicated by dashed boxes. Red arrows indicate areas of injury, including alveolar spaces filled with a mononuclear/neutrophilic infiltrates, cellular debris, and proteinaceous material. (C-H) The total number of neutrophils (C), protein content (D), and proinflammatory cytokines $(\mathbf{E}-\mathbf{H})$ in BAL fluids of indicated groups of mice. Data presented as mean $\pm \mathrm{SD}(n=3-5) .{ }^{*} P<0.05$ (ANOVA).

Further evidence of NLRP3 activation is provided by the finding that levels of IL-1 $\beta$ were elevated and caspase 1 was activated by LPS stimulation, and both of these responses were exacerbated by SIRT3 deficiency (Supplemental Figure 2). Moreover, the mitochondria-targeted antioxidant, MitoTEMPO, significantly

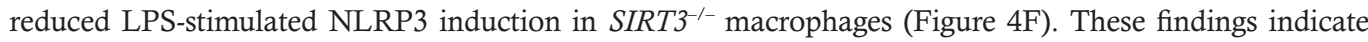
that deficiency of SIRT3 leads to enhanced macrophage proinflammatory activation, in part by activating the NLRP3 inflammasome. Our results also indicate that the viniferin, via a SIRT3-dependent mechanism, effectively reduces LPS-induced proinflammatory responses and inflammasome activation in macrophages.

SIRT3 activation modulates LPS-induced alterations in mitochondrial bioenergetics. Next, cellular bioenergetics were assessed in macrophages treated with LPS with/without viniferin. The effects of LPS on basal OCR were not significantly altered by viniferin (Figure 5, A and B); however, the decline in ATP-linked, maximal, and reserve capacity induced by LPS was significantly prevented by viniferin treatment (Figure 5, A and C-E). Importantly, the increased proton leak induced by LPS was also decreased by viniferin (Figure 5F), suggesting improved coupling of electron transport to ATP synthesis. No significant changes 

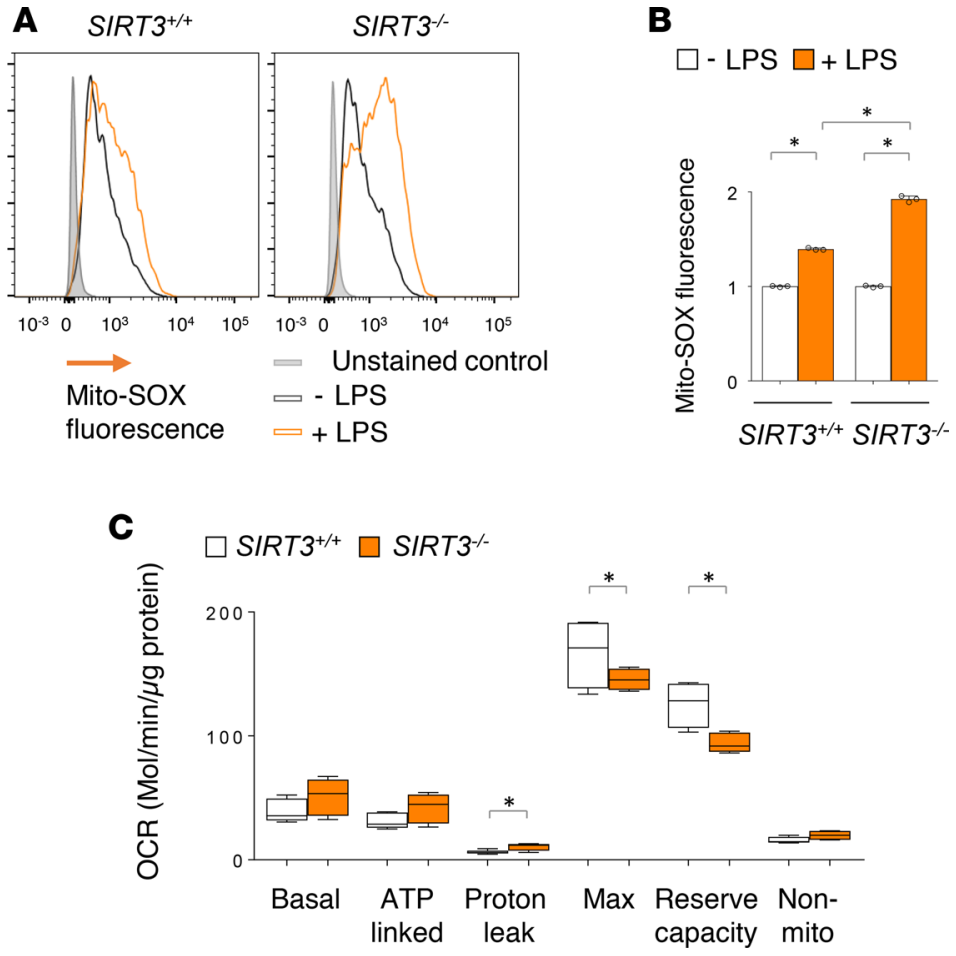

Figure 3. The effects of SIRT3 on macrophage mitochondrial ROS formation and bioenergetics. (A and B) Representative histograms (A) and quantitative analysis (B) of MitoSOX fluorescence in control or LPS-treated peritoneal

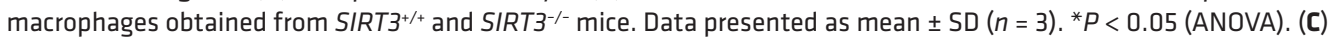
The oxygen consumption rate (OCR) in $S I R T 3^{+/+}$and $S I R T 3^{-/-}$macrophages. Indices of mitochondrial function include basal, ATP-linked, maximal, reserve capacity, proton leak, and non-mitochondrial oxygen consumption. Data presented as mean \pm SD $(n=3-6) .{ }^{*} P<0.05$ (Student's $t$ test).

with viniferin or LPS treatment were noted for non-mitochondrial respiration (Figure 5G). These results indicate that viniferin improves mitochondrial bioenergetics and prevents LPS-induced mitochondrial reprogramming. Based on the observed increase in basal, ATP-linked, and maximal OCR by viniferin in unstimulated (non-LPS) cells, we examined the potential for induction of mitochondrial biogenesis by this SIRT3 inducer; we found that viniferin upregulated the expression of mitochondrial transcription factor A (TFAM), a critical regulator of mitochondrial biogenesis (Supplemental Figure 4).

SIRT3 activation reduces the severity of endotoxin-induced ALI. Based on the observed beneficial effects of viniferin on macrophage mitochondrial bioenergetics and inflammatory responses, we next tested if viniferin confers protective effects in a mouse model of endotoxin-induced ALI. Mice were pretreated with viniferin $(120 \mathrm{mg} / \mathrm{kg}$, i.p.) once a day for 3 consecutive days followed by i.t. instillation of LPS ( $3 \mathrm{mg} / \mathrm{kg}$, i.t.). The control group received vehicle (DMSO and saline, 1:10, i.p.; or saline, i.t.). We found that viniferin diminished the severity of LPS-induced ALI, as evidenced by a lower neutrophil flux and reduced accumulation of debris in alveolar spaces, and overall preserved lung architecture (Figure 6, A and B). Viniferin also diminished pulmonary edema formation, as indicated by decreased amounts of protein in BAL fluids (Figure 6C). Western blot analysis of whole-lung homogenates revealed that viniferin significantly prevented the reduction in SIRT3 expression following LPS exposure (Figure 6, D and E). Consistent with experiments ex vivo (Figure 4C), viniferin significantly diminished production of several proinflammatory cytokines, including TNF- $\alpha$, MIP-2, and IL-6, in LPS-treated mice (Figure 7A). Furthermore, viniferin reduced accumulation of harmful DAMPs, including HMGB1 in BAL fluids of LPS-treated mice (Figure 7B). Similar to our findings obtained in ex vivo macrophages (Figure 4E), viniferin also diminished LPS-induced NLRP3 expression (whole-lung homogenates; Figure 7C). This was accompanied with lower production of inflammasome-dependent IL-1 $\beta$ (Figure 7D). These antiinflammatory and lung-protective

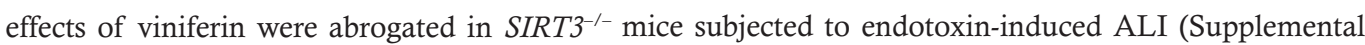
Figure 3, A-E). Together, these findings indicate that viniferin mediates protective effects in a mouse model of endotoxin-induced ALI through SIRT3, in association with macrophage deactivation. 
A

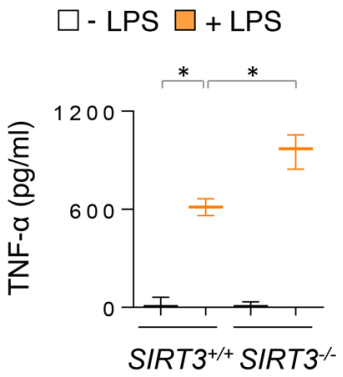

B

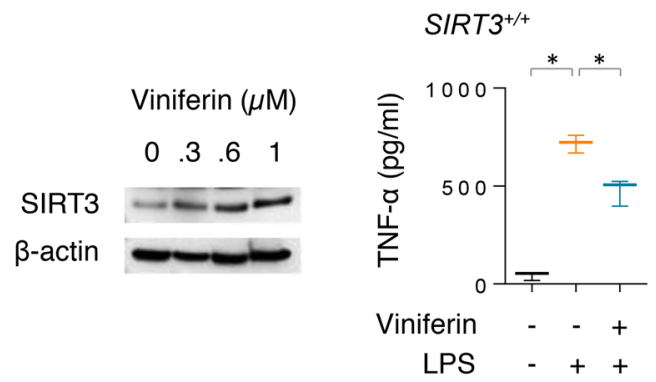

D

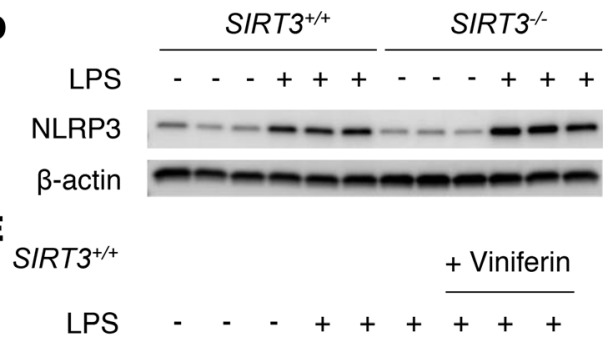

NLRP3

$\beta$-actin

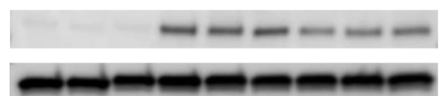

SIRT3

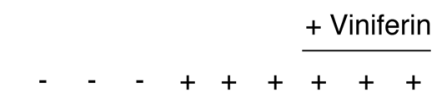

LPS

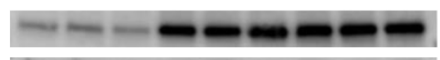

$\beta$-actin

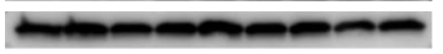

\section{F}

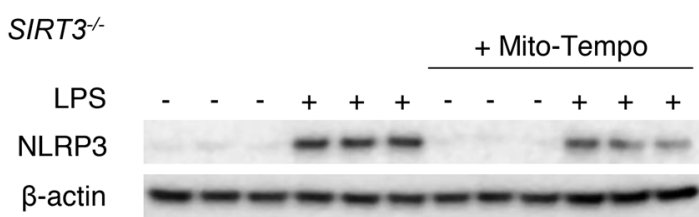

SIRT3-

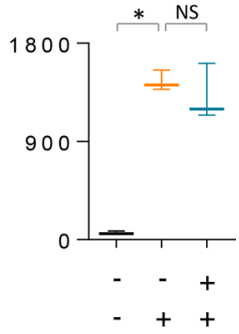

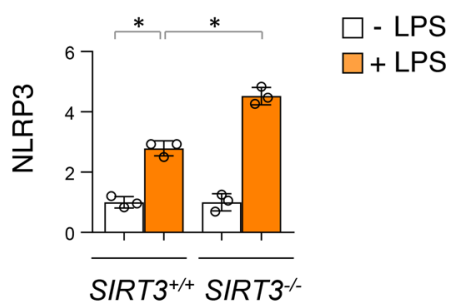

C

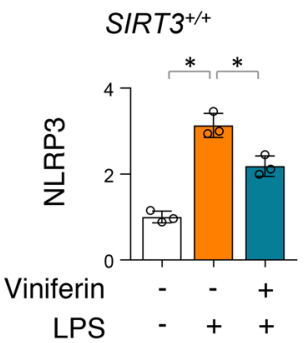

SIRT\%
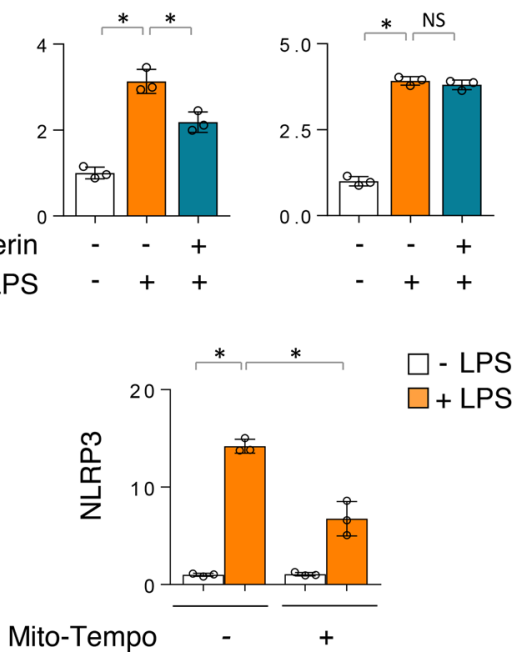

Figure 4. SIRT3 deficiency promotes macrophage proinflammatory activation. (A) Panels show the TNF- $\alpha$ levels in

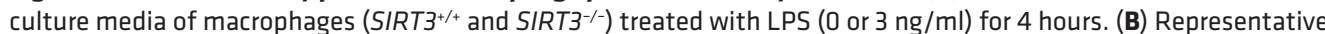
Western blots of SIRT3 and $\beta$-actin from macrophages treated with the indicated doses of viniferin for 24 hours. (C) TNF- $\alpha$ was measured in culture media from control, LPS, or LPS- and viniferin-treated macrophages $\left(S I R T 3^{+/+}\right.$and SIRT3-(-). (D) Representative Western blots and optical band densitometry of NLRP3 in wild-type and SIRT3-deficient macrophages. Cells were treated with LPS ( 0 or $3 \mathrm{ng} / \mathrm{ml}$ ) for 4 hours. (E) Representative NLRP3 immunoblot from macrophages $\left(S I R 3^{+/+}\right.$or SIRT3 $\left.{ }^{--}\right)$treated with LPS alone $(3 \mathrm{ng} / \mathrm{ml})$ or pretreated with viniferin $(1 \mu \mathrm{M}, 24$ hours) and then LPS for 4 hours. (F) Western immunoblots and quantitative analysis of NLRP3 in peritoneal macrophages (SIRT3 ${ }^{-/-}$) pretreated with MitoTEMPO ( 0 or $50 \mathrm{nM}, 18$ hours) and then LPS ( 0 or $3 \mathrm{ng} / \mathrm{ml}$ ) for an additional 4 hours. Data in $\mathbf{A}$ and C-F presented as mean $\pm \mathrm{SD}(n=3) .{ }^{*} P<0.05$ (ANOVA). NS, not significant.

\section{Discussion}

Despite significant improvements in the delivery of critical care, ALI/ARDS mortality remains high, with no approved pharmacologic therapies (10). While there are many reasons for lack of efficacy of experimental drugs, few approaches have targeted the metabolic reprogramming of immune cells. This study investigated the functional role of SIRT3, a mitochondrial $\mathrm{NAD}^{+}$-dependent deacetylase in a murine model of endotoxin-induced ALI. Our results support a regulatory role for SIRT3 in controlling the inflammatory response to LPS. This conclusion is based on the following observations: (a) decreased expression of SIRT3 in lung tissues following endotoxin-induced ALI; (b) SIRT3 ${ }^{-1-}$ mice 
A
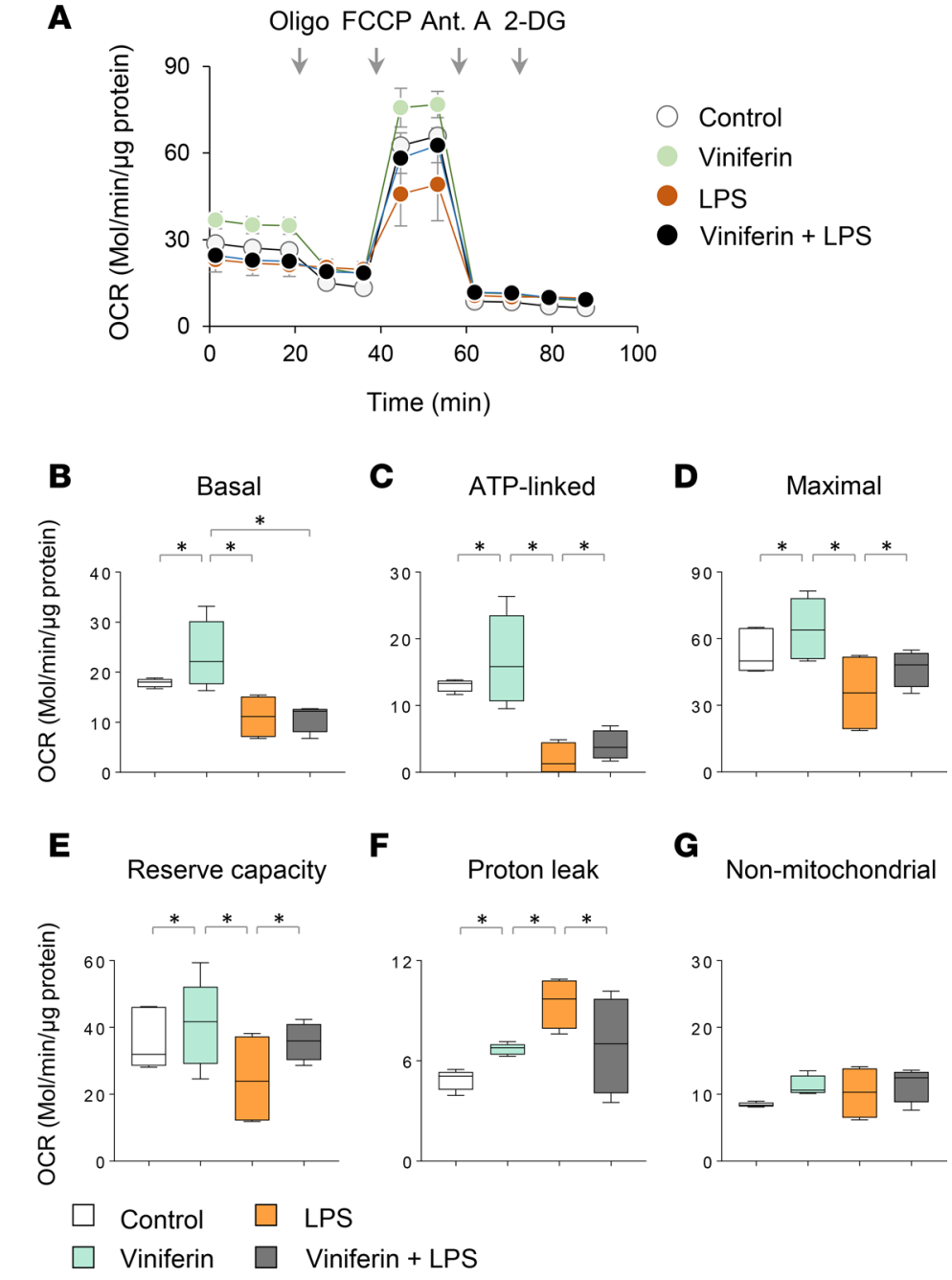

Figure 5. SIRT3 activation by viniferin modulates LPS-induced alterations in mitochondrial bioenergetics. (A) Representative traces showing the oxygen consumption rates (OCRs) in control and LPS-treated $(3 \mathrm{ng} / \mathrm{ml}$ ) macrophages. Oligo, oligomycin; FCCP, carbonyl cyanide-4-(trifluoromethoxy)phenylhydrazone; ant. A, antimycin A; 2-DC, 2-deoxyglucose. Cells were incubated with or without viniferin $(1 \mu \mathrm{M})$ for 8 hours and then incubated with LPS for an additional 16 hours. (B-C) Indices of mitochondrial function, including (B) basal, (C) ATP linked, (D) maximal, (E) reserve capacity, (F) proton leak, and (G) non-mitochondrial OCRs. Data presented as mean $\pm \operatorname{SEM}(n=4)$. ${ }^{*} P<0.05$ (ANOVA).

develop enhanced inflammatory responses and more severe ALI, compared with wild-type mice; (c) increased production of proinflammatory cytokines including TNF- $\alpha$, MIP2, and IL-6 in association

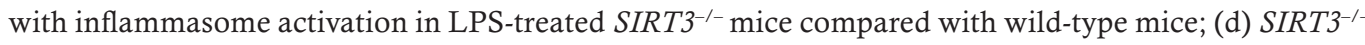
deficiency in macrophages increases mitochondrial ROS, which activates the NLRP3 inflammasome in response to LPS; and (e) a pharmacological modulator that increases SIRT3 levels reduces the severity of endotoxin-induced ALI, but this protective effect is lost in SIRT3-deficient mice. These findings provide a critical link between SIRT3 deficiency, macrophage bioenergetic reprogramming, and an enhanced macrophage proinflammatory response that contributes to the severity of ALI (Figure 8).

This is the first study, to our knowledge, that demonstrates a critical role for SIRT3 in regulating the inflammatory responses to LPS-induced ALI. Previous reports have implicated SIRT3 deficiency in cardiac hypertrophy, organ fibrosis, neurodegenerative diseases, cancer, and acute kidney injury (AKI) (27, 33-37). SIRT3-knockout mice develop tissue fibrosis of multiple organs, including the lungs, with aging (38); in our studies, we did not detect lung phenotypic changes at baseline in mice at 2 months of age. SIRT3 levels were significantly decreased in the cerebral cortex of subjects with Alzheimer's disease and associated with enhanced neuronal damage in vitro (25). Analogous to our findings of decreased SIRT3 expression in ALI, diminished SIRT3 expression in kidney tissue was observed in a murine model of cisplatin-induced AKI 


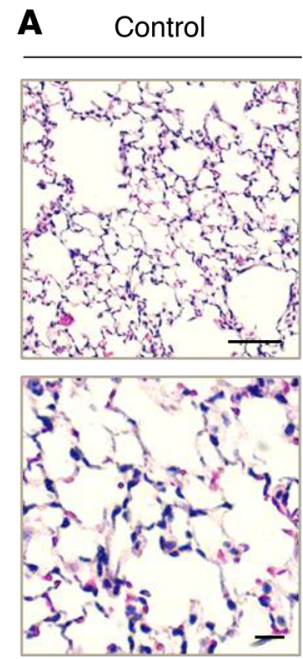

B

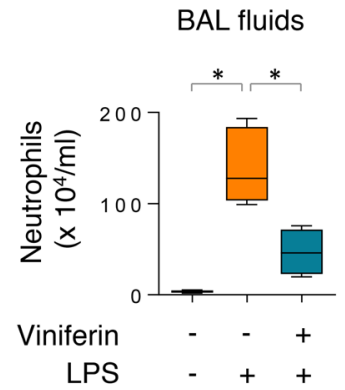

D

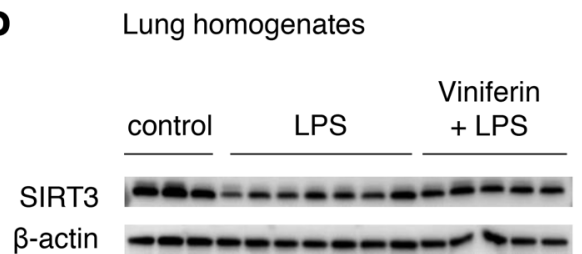

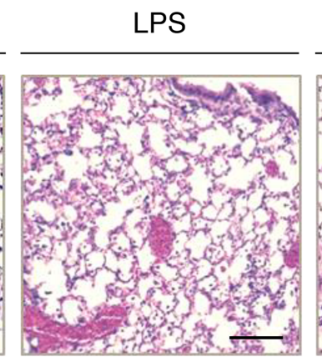
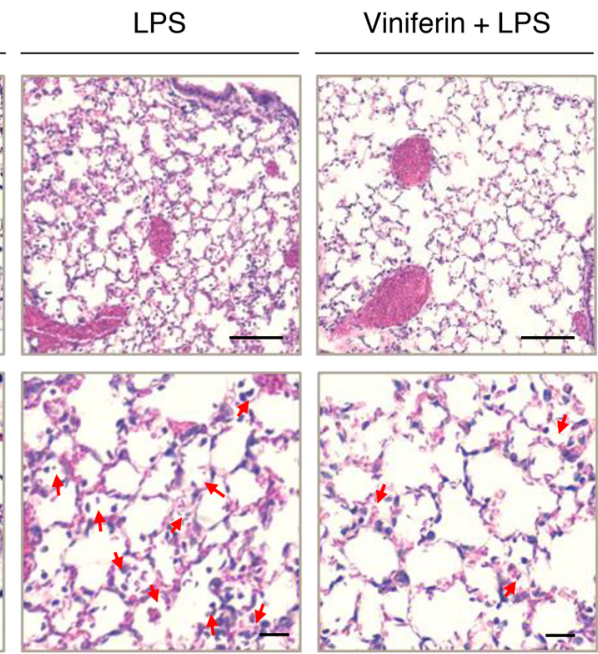

C

BAL fluids

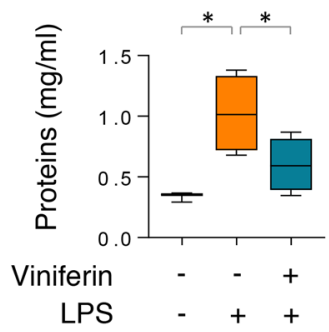

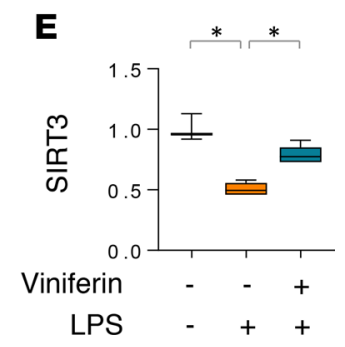

Figure 6. Viniferin diminishes the severity of endotoxin-induced ALI. (A) Representative images show H\&E staining of lung sections from control (saline) and mice subjected to LPS ( $3 \mathrm{mg} / \mathrm{kg}$, i.t.) for 24 hours. Mice received viniferin (0 or $120 \mathrm{mg} / \mathrm{kg}$, i.p.) once a day for 3 consecutive days prior to LPS exposure. Scale bars: $100 \mu \mathrm{m}$ (upper panel) and $10 \mu \mathrm{m}$ (lower panel). (B and $\mathbf{C}$ ) The number of neutrophils (B) and the amount of protein (C) in BAL fluids of control, LPS alone, or viniferin- and LPS-treated mice. (D and E) Representative Western blots and quantitative analysis of SIRT3 in lung homogenates of indicated groups of mice. Data presented as mean $\pm \operatorname{SD}(n=3-7) .{ }^{*} P<0.05$ (ANOVA).

and in sepsis-induced AKI $(35,37)$. A previous study reported reduced SIRT3 in LPS-induced pericyte loss and enhanced vascular leak, while SIRT3 overexpression reversed these effects (39). Protective effects of SIRT3 on hyperoxia-induced ALI have been linked to enzymatic activation of SOD2 (40). Our studies did demonstrate a significant reduction in total SOD2, without an apparent change in its relative acetylated (activated) state, in a bacterial pneumonia model. However, our studies do implicate macrophage reprogramming and mitochondrial superoxide formation by SIRT3 as a critical mediator of the heightened inflammation and severity of lung injury in an animal model of endotoxin-induced lung injury.

Our data indicate that the levels of SIRT3 expression/activation in macrophages can function as a rheostat to control proinflammatory NLRP3 inflammasome activation. NLRP3 inflammasome activation has been reported in kidney tissues of SIRT3-deficient mice following AKI (37). Prolonged fasting, which is known to induce SIRT3 activity in different cell types (41-43), was found to suppress NLRP3 inflammasome activation via the activation of SOD2 (44). Our results indicate that mitochondrial SIRT3 expression/activation is linked to mitochondrial bioenergetics and the control of macrophage inflammatory responses. Further studies are 

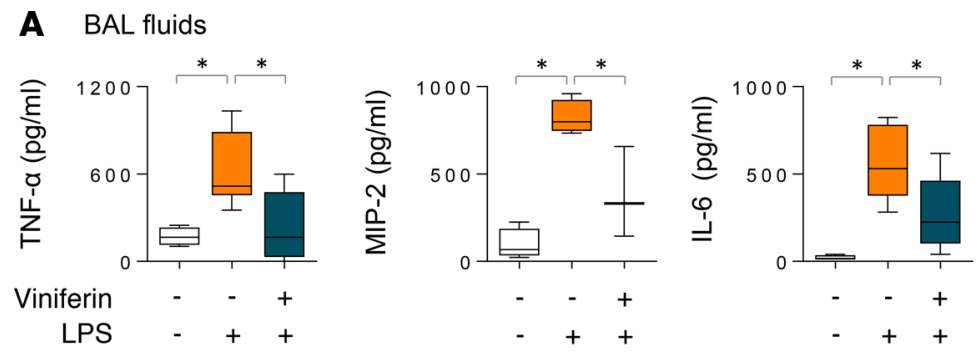

B
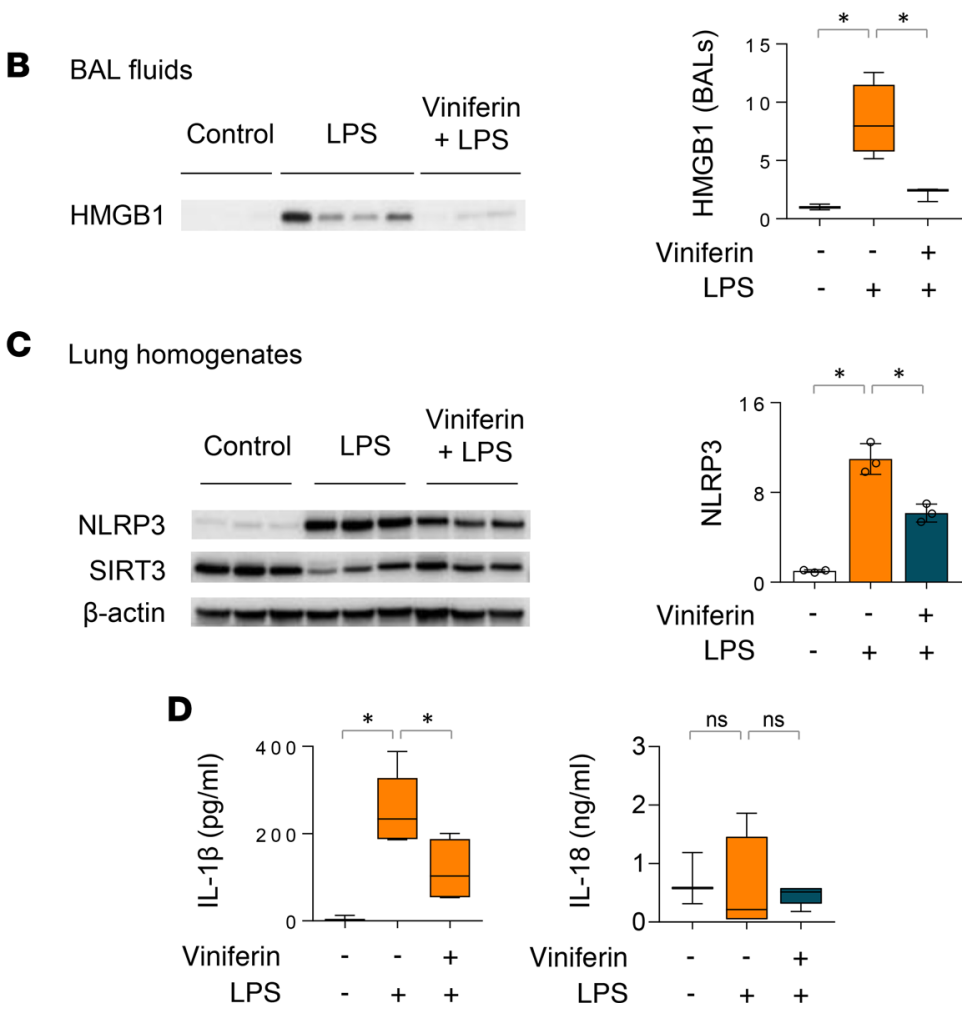

Figure 7. Viniferin reduces lung inflammation in mice subjected to LPS-induced ALI. (A) Panels show TNF- $\alpha$, MIP-2, and IL-6 levels in BAL fluids of control, LPS-treated, or mice treated with a combination of viniferin and LPS (depicted in Figure 6). (B and C) Representative Western blots and optical band densitometry of (B) HMGB1 in BAL fluids or (C) NLRP3 and SIRT3 in whole-lung homogenates from indicated groups of mice. (D) IL-1 $\beta$ and IL-18 cytokine levels in BAL fluids from mice treated as indicated in Figure 6. Data presented as mean \pm SD $(n=3-5)$. ${ }^{*} P<0.05 ;$ ns, not significant (ANOVA).

warranted to determine the precise mechanisms by which SIRT3 modulates the function of immune cells. Previous studies suggest that SIRT3 modulates mitochondrial function and integrity by reducing dynamin-related protein-dependent fission, mitochondrial membrane depolarization, and mitophagy (35).

We further investigated whether pharmacological activation of SIRT3 can mitigate the severity of endotoxin-induced ALI. We utilized viniferin, a specific inducer/activator of SIRT3 (30), to provide proof-of-concept that SIRT3 upregulation by a pharmacologic approach can mediate protective effects. Our data indicate that in vivo SIRT3 induction with viniferin treatment reduces the severity of LPS-induced ALI, as evidenced by diminished neutrophilic influx, lung permeability, HMGB1 release, and NLRP3 activation; importantly, viniferin failed to mediate these lung protective effects in SIRT3-deficient mice. Although our studies implicate deficient SIRT3 in regulating macrophage proinflammatory responses, which are modulated by viniferin, it is possible that some of the beneficial effects of viniferin may be related to its effects on non-macrophage cell populations. Previous studies have shown that attenuation of proinflammatory responses by similar approaches is not associated with impairment in macrophage antimicrobial functions $(16,45)$. On the contrary, it has been suggested that mitochondrial biogenesis and normalization of redox homeostasis may improve immune responses (46-48). Mitochondrial dysfunction 


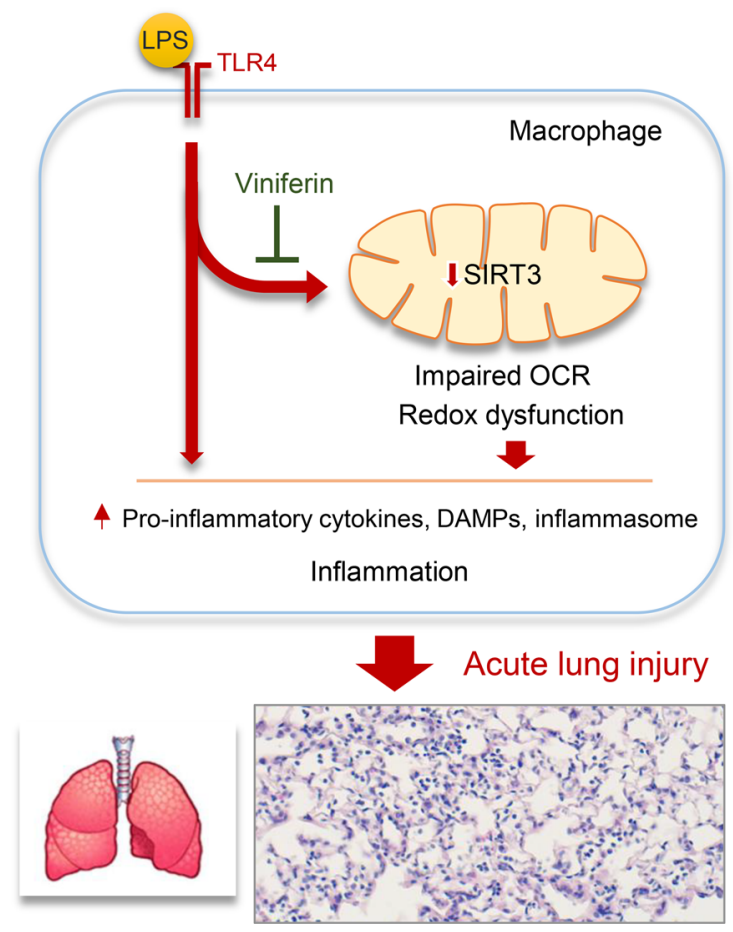

Figure 8. Proposed mechanism of SIRT3 deficiency in exacerbation of endotoxin-induced ALI. ALI induces SIRT3 deficiency in lungs of LPS-treated mice in association with increased inflammation and worsened ALI. Viniferin modulates mitochondrial bioenergetics, production of proinflammatory cytokines and DAMPs, as well as inflammasome activation. Pretreatment of mice with viniferin reduces inflammation and severity of endotoxin-induced ALI.

has been linked to sepsis-induced organ failure associated with high morbidity and mortality rates (11, 13); thus, strategies that preserve mitochondrial function and control inflammation without diminishing host defense functions could be an effective therapeutic approach in sepsis and multiorgan failure.

\section{Methods}

Animal studies. SIRT3 wild-type (129S1/SvImJ; The Jackson Laboratory, 002448) and SIRT3-knockout (129-Sirt3 ${ }^{\text {tml.1Fwa }} / \mathrm{J}$; The Jackson Laboratory, 012755) mice were used for the study. The mice were kept on a 12-hour light/dark cycle with free access to food and water. Endotoxin-induced ALI was initiated by i.t. instillation of LPS, which is a clinically relevant model for moderate lung injury. ALI was induced in 2-monthold SIRT3 $^{+/+}$and SIRT3 ${ }^{-/}$mice by i.t. administration of $3 \mathrm{mg} / \mathrm{kg}$ E. coli 0111:B4 LPS in $50 \mu \mathrm{l}$ of saline as previously described (49). Briefly, mice were anesthetized with a mixture of isoflurane $(2 \%, \mathrm{v} / \mathrm{v})$ and oxygen vapor. The tongue was gently pulled out from mouth and LPS in saline (50 $\mu$ l) was instilled into the distal part of the oropharynx. Mice were euthanized 24 hours after LPS administration. To investigate effects of viniferin in endotoxin-induced ALI, $S I R T 3^{+/+}$and $S I R T 3^{-/}$mice were pretreated with viniferin $(120 \mathrm{mg} / \mathrm{kg}$, i.p.) once a day for 2 days and then 2 hours prior to i.t. instillation of LPS. Mice were euthanized 24 hours after LPS administration. Lungs were harvested for downstream analysis. BAL was obtained by cannulating the trachea with a blunt 20-gauge needle and then lavaging the lungs 3 times with $1 \mathrm{ml}$ of PBS.

A mouse model of bacterial pneumonia. P. aeruginosa strain K suspension in PBS $\left(2.5 \times 10^{7} /\right.$ mouse, $\left.40 \mu \mathrm{l}\right)$ or PBS alone (control, $40 \mu \mathrm{l}$ ) was deposited into the pharynx followed by bacterial aspiration into the lungs, as previously described (50). Lung homogenates were prepared 4 hours after $P$. aeruginosa instillation and serial dilutions used to determine colony-forming units (CFUs) on agar plates.

Reagents and antibodies. LPS derived from E. coli 0111:B4 (catalog L2630) and $\beta$-actin antibody (cata$\log$ A1978) were purchased from Sigma-Aldrich. Viniferin was obtained from FUJIFILM Wako Chemicals USA Corporation. MitoTEMPO was purchased from Cayman Chemical. Antibodies against SIRT3 (catalog 5490), NLRP3 (catalog 15101), and cleaved caspase-1 (catalog67314) were obtained from Cell Signaling Technology. Anti-HMGB1 (catalog18256) and -MnSOD (acetyl K68) (catalog ab137037) were from Abcam. COX IV antibody (catalog MAB6980) and antibody against IL-1ß (catalogAF-401-NA) were 
purchased from R\&D Systems. TFAM antibody was purchased from Gene Tex (catalog GTX103231). RPMI-1640 was purchased from Invitrogen.

Macrophage isolation and culture. Peritoneal macrophages were isolated as described previously (51). Macrophages were elicited in mice by using i.p. injection of Brewer thioglycollate. Cells were collected 3 days after i.p. injection of thioglycollate. Macrophages were then cultured in 12 -well plates $\left(5 \times 10^{5}\right.$ cells/ well) in RPMI-1640 media containing 5\% fetal calf serum (HyClone Laboratories), 100 units/ml penicillin, $100 \mu \mathrm{g} / \mathrm{ml}$ streptomycin, $1.25 \mu \mathrm{g} / \mathrm{ml}$ amphotericin $\mathrm{B}$, at $37^{\circ} \mathrm{C}, 5 \% \mathrm{CO}_{2}, 95 \%$ air.

Isolation of mitochondria. Lung mitochondria were isolated according to standard procedures with minor modifications (52). Lung homogenates were incubated in STE buffer containing sucrose (250 $\mathrm{mM})$, Tris pH 7.4 (10 mM), and ethyleneglycol-bis-( $\beta$-aminoethyl ether)- $N, N^{\prime}$-tetraacetic acid (EGTA) (2 mM), followed by centrifugation $(1,000 \mathrm{~g})$ for 3 minutes at $4^{\circ} \mathrm{C}$. Supernatants were then collected and centrifuged $(10,000 \mathrm{~g})$ for 10 minutes at $4^{\circ} \mathrm{C}$. The resultant pellets were suspended in STE buffer and centrifuged $(10,000 \mathrm{~g})$ for 10 minutes at $4^{\circ} \mathrm{C}$. The pink and light-brown pellets surrounding the dark-brown center were aspirated and the remaining pellet suspended in STE buffer $(1 \mathrm{ml})$ and centrifuged $(10,000 \mathrm{~g})$ for 15 minutes at $4^{\circ} \mathrm{C}$. Finally, the cell pellet was suspended in RIPA buffer and stored at $-80^{\circ} \mathrm{C}$.

Lung histology and immunofluorescence staining. Mouse lungs were inflated with $1 \mathrm{ml}$ of $10 \%$ paraformaldehyde/PBS solution and embedded in paraffin. Immunofluorescence staining was performed as described previously (53). Images were acquired using a Nikon A1 Laser Confocal Microscope at the University of Alabama at Birmingham (UAB) High Resolution Imaging Facility. The levels of fluorescence were quantified and displayed as 2-dimensional scattergrams using Hamamatsu software (Hamamatsu Corporation).

Detection of ROS formation. The levels of superoxide generation were measured by MitoSOX probe (Thermo Fisher Scientific) in conjunction with flow cytometry, according to the manufacturer's instructions. In brief, peritoneal macrophages were grown in monolayer, treated with $300 \mathrm{ng} / \mathrm{ml}$ LPS for 3 hours, and then with $5 \mathrm{mM}$ ATP for 15 minutes and incubated with the fluorescent probe MitoSOX $(5 \mu \mathrm{M})$ for 15 minutes at $37^{\circ} \mathrm{C}$. Cells were washed with PBS, detached from cultured plates with trypsin, and then fluorescence intensity measured using flow cytometry and FlowJo analytical software version 10 (Tree Star).

Measurement of cytokines. ELISA was used to measure cytokine levels in culture media and BAL fluids, as previously described (50). Levels of TNF- $\alpha$, MIP-2, IL-6, IL1- $\beta$, and IL-18 were determined using commercially available ELISA kits (R\&D Systems) according to the manufacturer's instructions.

Western blot analysis. Cell lysates were prepared in RIPA buffer supplemented with protease inhibitors and subjected to SDS-PAGE under reducing conditions, and Western immunoblotting was performed as previously described (53). BAL fluids $(30 \mu \mathrm{l})$ were mixed with Laemmli sample buffer (Boston BioProducts) and boiled for 5 minutes followed by Western blot analysis with anti-HMGB1 antibody. Immunoblots were imaged using an Amersham Biosciences 600 Imager (GE Healthcare). Densitometric analyses were performed using AlphaEaseFC software (Alpha Innotech) and ImageJ software (http://imagej.nih.gov/ij/).

Real-time PCR. Total RNA was isolated from lung tissues using the RNeasy Mini kit (Qiagen) and reverse transcribed using iScript Reverse Transcription SuperMix for RT-quantitative PCR (Bio-Rad). Realtime PCRs were performed using SYBR Green PCR Master Mix (Life Technologies) and gene-specific primer pairs for SIRT3 and 18S rRNA. Primer sequences for SIRT3: forward 5'-CATATGGGCTGATGTGATGG-3' and reverse 5'-GCCATATGGAGTAGGAACCTTG-3'. For 18S: forward 5'-GTCTGCCCTATCAACTTTCG-3', reverse 5'-ATGTGGTAGCCGTTTCTCAG-3'. Real-time PCR and analysis were performed as described previously (53).

Mitochondrial bioenergetics. Macrophages were cultured on XFe96 plates using RPMI-1640 media containing $5 \%$ fetal calf serum for 24 hours prior to the assay. On the next day, cells were pretreated viniferin $(1 \mu \mathrm{M}$ for 6-8 hours) and then incubated with or without LPS (3 ng/ml) overnight. Cells were washed in XF assay buffer (DMEM with $1 \mathrm{mM}$ pyruvate, $5.5 \mathrm{mM}$ D-glucose, $4 \mathrm{mM} \mathrm{L}$-glutamine, $\mathrm{pH} 7.4$ ) and incubated in XF assay buffer for 30 to 60 minutes before the assay. Bioenergetics of macrophages were determined using the XF24 analyzer from Seahorse Bioscience, which measures $\mathrm{O}_{2}$ consumption and proton production $(\mathrm{pH})$ in cells, performed and interpreted as described previously $(54,55)$. ATP-linked OCR and proton leak were determined by injecting oligomycin $(0.5 \mu \mathrm{M})$, an inhibitor of ATP synthase. FCCP $(1.5 \mu \mathrm{M})$, an uncoupler of the electron transport chain, was used to determine maximal respiration rate. The complete inhibition of mitochondrial electron transport was achieved using antimycin $\mathrm{A}(10 \mu \mathrm{M})$, an inhibitor of complex III. The OCR determined after antimycin A $(10 \mu \mathrm{M})$ injection is attributable to non-mitochondrial oxygen consumption. Mitochondrial basal respiration, proton leak, and the maximal respiration were calculated after correcting for non-mitochondrial OCR. 
Statistical analysis. Data are expressed as mean \pm SD or SEM. Statistical analysis was performed using Student's $t$ test (1 tailed) or 1-way ANOVA to evaluate differences between groups. A value of $P<0.05$ was considered significant. Analyses were performed using GraphPad Prism software (version 6.04).

Study approval. All experiments were conducted in accordance with approved protocols by the University of Alabama at Birmingham, Institutional Animal Care and Use Committee (IACUC).

\section{Author contributions}

DK, JWZ, and VJT conceived and designed the study. DK, ARK, NBB, WL, EJB, BC, VDU, JWZ, and VJT performed experiments and data analysis and interpretation. DK, JWZ, and VJT drafted the manuscript.

\section{Acknowledgments}

This work was supported by NIH grants P01 HL114470 and R01 AG046210 (to VJT), US Department of Defense grant W81XWH-17-1-0577 (to JWZ), and a US Department of Veterans Affairs Merit Award I01BX003056 (to VJT).

Address correspondence to: Victor J. Thannickal or Jaroslaw W. Zmijewski, Division of Pulmonary, Allergy, and Critical Care Medicine, Department of Medicine, University of Alabama at Birmingham, Birmingham, AL 35294, USA. Phone: 205.975.6376; Email: vthannickal@uabmc.edu (V. Thannickal). Phone: 205.934.7793; Email: zmijewsk@uab.edu (J. Zmijewski).

1. Rezoagli E, Fumagalli R, Bellani G. Definition and epidemiology of acute respiratory distress syndrome. Ann Transl Med. 2017;5(14):282.

2. Mason C, Dooley N, Griffiths M. Acute respiratory distress syndrome. Clin Med (Lond). 2016;16(Suppl 6):s66-s70

3. Wang H, et al. HMG-1 as a late mediator of endotoxin lethality in mice. Science. 1999;285(5425):248-251.

4. Villar J, et al. The ALIEN study: incidence and outcome of acute respiratory distress syndrome in the era of lung protective ventilation. Intensive Care Med. 2011;37(12):1932-1941.

5. Matthay MA, McAuley DF, Ware LB. Clinical trials in acute respiratory distress syndrome: challenges and opportunities. Lancet Respir Med. 2017;5(6):524-534.

6. Delano MJ, Ward PA. Sepsis-induced immune dysfunction: can immune therapies reduce mortality? J Clin Invest. 2016;126(1):23-31.

7. Levy JH. The human inflammatory response. J Cardiovasc Pharmacol. 1996;27 Suppl 1:S31-S37.

8. Lawrence T, Willoughby DA, Gilroy DW. Anti-inflammatory lipid mediators and insights into the resolution of inflammation Nat Rev Immunol. 2002;2(10):787-795.

9. Fein AM, Calalang-Colucci MG. Acute lung injury and acute respiratory distress syndrome in sepsis and septic shock. Crit Care Clin. 2000;16(2):289-317.

10. Padkin A, Goldfrad C, Brady AR, Young D, Black N, Rowan K. Epidemiology of severe sepsis occurring in the first 24 hrs in intensive care units in England, Wales, and Northern Ireland. Crit Care Med. 2003;31(9):2332-2338.

11. Brealey D, et al. Association between mitochondrial dysfunction and severity and outcome of septic shock. Lancet. 2002;360(9328):219-223.

12. Japiassú AM, et al. Bioenergetic failure of human peripheral blood monocytes in patients with septic shock is mediated by reduced F1Fo adenosine-5'-triphosphate synthase activity. Crit Care Med. 2011;39(5):1056-1063.

13. Singer M. The role of mitochondrial dysfunction in sepsis-induced multi-organ failure. Virulence. 2014;5(1):66-72.

14. Arulkumaran N, et al. Mitochondrial function in sepsis. Shock. 2016;45(3):271-281.

15. Mannam P, et al. MKK3 regulates mitochondrial biogenesis and mitophagy in sepsis-induced lung injury. Am J Physiol Lung Cell Mol Physiol. 2014;306(7):L604-L619.

16. Bone NB, Liu Z, Pittet JF, Zmijewski JW. Frontline Science: D1 dopaminergic receptor signaling activates the AMPK-bioenergetic pathway in macrophages and alveolar epithelial cells and reduces endotoxin-induced ALI. J Leukoc Biol. 2017;101(2):357-365.

17. Ciarlo E, et al. Sirtuin 2 deficiency increases bacterial phagocytosis by macrophages and protects from chronic staphylococcal infection. Front Immunol. 2017;8:1037.

18. Mesquita I, et al. Exploring NAD ${ }^{+}$metabolism in host-pathogen interactions. Cell Mol Life Sci. 2016;73(6):1225-1236.

19. Houtkooper RH, Pirinen E, Auwerx J. Sirtuins as regulators of metabolism and healthspan. Nat Rev Mol Cell Biol. 2012;13(4):225-238.

20. Finkel T, Deng CX, Mostoslavsky R. Recent progress in the biology and physiology of sirtuins. Nature. 2009;460(7255):587-591.

21. Duan WJ, et al. A SIRT3/AMPK/autophagy network orchestrates the protective effects of trans-resveratrol in stressed peritoneal macrophages and RAW 264.7 macrophages. Free Radic Biol Med. 2016;95:230-242.

22. Xu H, Hertzel AV, Steen KA, Bernlohr DA. Loss of fatty acid binding protein $4 / \mathrm{aP} 2$ reduces macrophage inflammation through activation of SIRT3. Mol Endocrinol. 2016;30(3):325-334.

23. Sheng S, Kang Y, Guo Y, Pu Q, Cai M, Tu Z. Overexpression of Sirt3 inhibits lipid accumulation in macrophages through mitochondrial IDH2 deacetylation. Int J Clin Exp Pathol. 2015;8(8):9196-9201.

24. Giralt A, Villarroya F. SIRT3, a pivotal actor in mitochondrial functions: metabolism, cell death and aging. Biochem J. 2012;444(1):1-10. 
25. Schwer B, North BJ, Frye RA, Ott M, Verdin E. The human silent information regulator (Sir)2 homologue hSIRT3 is a mitochondrial nicotinamide adenine dinucleotide-dependent deacetylase. J Cell Biol. 2002;158(4):647-657.

26. Onyango P, Celic I, McCaffery JM, Boeke JD, Feinberg AP. SIRT3, a human SIR2 homologue, is an NAD-dependent deacetylase localized to mitochondria. Proc Natl Acad Sci USA. 2002;99(21):13653-13658.

27. Lee J, et al. SIRT3 deregulation is linked to mitochondrial dysfunction in Alzheimer's disease. Aging Cell. 2018;17(1).

28. Rangarajan S, Bernard K, Thannickal VJ. Mitochondrial dysfunction in pulmonary fibrosis. Ann Am Thorac Soc. 2017;14(Supplement_5):S383-S388.

29. Domenici-Lombardo L, et al. Evolution of endotoxin induced acute lung injury in the rat. Int J Exp Pathol. 1995;76(5):381-390.

30. Fu J, et al. trans-(-)-ع-Viniferin increases mitochondrial sirtuin 3 (SIRT3), activates AMP-activated protein kinase (AMPK), and protects cells in models of Huntington Disease. J Biol Chem. 2012;287(29):24460-24472.

31. Chung EY, et al. alpha-Viniferin suppresses the signal transducer and activation of transcription-1 (STAT-1)-inducible inflammatory genes in interferon-gamma-stimulated macrophages. J Pharmacol Sci. 2010;112(4):405-414.

32. Bauernfeind FG, et al. Cutting edge: NF-kappaB activating pattern recognition and cytokine receptors license NLRP3 inflammasome activation by regulating NLRP3 expression. J Immunol. 2009;183(2):787-791.

33. Sundaresan NR, Gupta M, Kim G, Rajamohan SB, Isbatan A, Gupta MP. Sirt3 blocks the cardiac hypertrophic response by augmenting Foxo3a-dependent antioxidant defense mechanisms in mice. J Clin Invest. 2009;119(9):2758-2771.

34. Akamata K, et al. SIRT3 is attenuated in systemic sclerosis skin and lungs, and its pharmacologic activation mitigates organ fibrosis. Oncotarget. 2016;7(43):69321-69336.

35. Morigi M, et al. Sirtuin 3-dependent mitochondrial dynamic improvements protect against acute kidney injury. J Clin Invest. 2015;125(2):715-726.

36. Finley LW, et al. SIRT3 opposes reprogramming of cancer cell metabolism through HIF1 $\alpha$ destabilization. Cancer Cell. 2011;19(3):416-428

37. Zhao WY, Zhang L, Sui MX, Zhu YH, Zeng L. Protective effects of sirtuin 3 in a murine model of sepsis-induced acute kidney injury. Sci Rep. 2016;6:33201.

38. Sundaresan NR, et al. SIRT3 blocks aging-associated tissue fibrosis in mice by deacetylating and activating glycogen synthase kinase 3ß. Mol Cell Biol. 2015;36(5):678-692.

39. Zeng H, He X, Tuo QH, Liao DF, Zhang GQ, Chen JX. LPS causes pericyte loss and microvascular dysfunction via disruption of Sirt3/angiopoietins/Tie-2 and HIF-2 $\alpha$ /Notch3 pathways. Sci Rep. 2016;6:20931.

40. Tian YG, Zhang J. Protective effect of SIRT3 on acute lung injury by increasing manganese superoxide dismutase-mediated antioxidation. Mol Med Rep. 2018;17(4):5557-5565.

41. Hebert AS, et al. Calorie restriction and SIRT3 trigger global reprogramming of the mitochondrial protein acetylome. Mol Cell. 2013;49(1):186-199.

42. Hirschey MD, et al. SIRT3 regulates mitochondrial fatty-acid oxidation by reversible enzyme deacetylation. Nature. 2010;464(7285):121-125.

43. Someya S, et al. Sirt3 mediates reduction of oxidative damage and prevention of age-related hearing loss under caloric restriction. Cell. 2010;143(5):802-812.

44. Traba J, et al. Prolonged fasting suppresses mitochondrial NLRP3 inflammasome assembly and activation via SIRT3-mediated activation of superoxide dismutase 2. J Biol Chem. 2017;292(29):12153-12164.

45. Tran M, et al. PGC-1 $\alpha$ promotes recovery after acute kidney injury during systemic inflammation in mice. J Clin Invest. 2011;121(10):4003-4014.

46. Perico L, et al. Human mesenchymal stromal cells transplanted into mice stimulate renal tubular cells and enhance mitochondrial function. Nat Commun. 2017;8(1):983.

47. Walker MA, Volpi S, Sims KB, Walter JE, Traggiai E. Powering the immune system: mitochondria in immune function and deficiency. J Immunol Res. 2014;2014:164309.

48. Weinberg SE, Sena LA, Chandel NS. Mitochondria in the regulation of innate and adaptive immunity. Immunity. 2015;42(3):406-417.

49. Zhao X, et al. Activation of AMPK attenuates neutrophil proinflammatory activity and decreases the severity of acute lung injury. Am J Physiol Lung Cell Mol Physiol. 2008;295(3):L497-L504.

50. Zmijewski JW, et al. Mitochondrial respiratory complex I regulates neutrophil activation and severity of lung injury. Am J Respir Crit Care Med. 2008;178(2):168-179.

51. Bae HB, et al. AMP-activated protein kinase enhances the phagocytic ability of macrophages and neutrophils. FASEB J. 2011;25(12):4358-4368.

52. Frezza C, Cipolat S, Scorrano L. Organelle isolation: functional mitochondria from mouse liver, muscle and cultured fibroblasts. Nat Protoc. 2007;2(2):287-295.

53. Hecker L, et al. Reversal of persistent fibrosis in aging by targeting Nox4-Nrf2 redox imbalance. Sci Transl Med. 2014;6(231):231ra47.

54. Dranka BP, et al. Assessing bioenergetic function in response to oxidative stress by metabolic profiling. Free Radic Biol Med. 2011;51(9):1621-1635.

55. Chacko BK, et al. Methods for defining distinct bioenergetic profiles in platelets, lymphocytes, monocytes, and neutrophils, and the oxidative burst from human blood. Lab Invest. 2013;93(6):690-700. 